Pacific

Journal of

Mathematics

MANIFOLDS WITH 2-NONNEGATIVE RICCI OPERATOR

Martha P. Dussan and Maria Helena Noronha 


\title{
MANIFOLDS WITH 2-NONNEGATIVE RICCI OPERATOR
}

\author{
Martha P. Dussan and Maria Helena Noronha
}

\begin{abstract}
In this paper we study compact manifolds with 2-nonnegative Ricci operator, assuming that their Weyl operator satisfies certain conditions which generalize conformal flatness. As a consequence, we obtain that such manifolds are either locally symmetric or their Betti numbers between 2 and $n-2$ vanish. We also study the topology of compact hypersurfaces with 2-nonnegative Ricci operator.
\end{abstract}

\section{Introduction.}

One of the most powerful methods for studying the Betti numbers of compact manifolds is the Bochner technique. This technique is used in the context of manifolds with some type of curvature condition which will imply that harmonic forms are parallel. For 2-forms, this fact is implied by the nonnegativity of the Weitzenböck operator. In dimension four the nonnegativity of the Weitzenböck operator is equivalent (see for instance [14]) to the nonnegativity of the isotropic curvature, a notion introduced by MicallefMoore ([11]) to study stability of harmonic 2-spheres. In that paper, the authors also point out that conformally flat manifolds with nonnegative scalar curvature have nonnegative isotropic curvature. Actually, denoting the Weitzenböck operator by $\mathcal{Q}_{2}$, they show that a necessary and sufficient condition for the nonnegativity of $\mathcal{Q}_{2}$ is $-\mathcal{W}+S / 6 \geq 0$, where $S$ is the scalar curvature and $\mathcal{W}$ is the operator induced by the Weyl tensor on the space of 2 -forms $\Lambda^{2}\left(T_{x} M\right)$. The condition above follows from the fact that, in dimension 4, the isotropic curvature (and hence the Weitzenböck operator) does not depend on the traceless Ricci tensor.

In dimensions greater than 4, conformal flatness and the nonnegativity of the scalar curvature do not imply nonnegative isotropic curvature, as can be seen through the conformally flat hypersurfaces constructed in [10] which have $S \geq 0$ but some isotropic curvatures are negative. The same examples of conformally flat manifolds show that for $n>4, S \geq 0$ does not imply $\mathcal{Q}_{2} \geq 0$.

The role of the Ricci tensor in the study of the isotropic curvature and the Weitzenböck operator for dimensions $n>4$ is not yet clear. It turns out (see below) that the condition $-\mathcal{W}+S / 6 \geq 0$ used for 4-manifolds generalizes to $-\mathcal{W}+S /[(n-2)(n-1)] \geq 0$ and this paper searches for hypotheses on 
the Ricci tensor that together with $-\mathcal{W}+S /[(n-2)(n-1)] \geq 0$ imply that $\mathcal{Q}_{2} \geq 0$. Our first result in this paper is the following:

Theorem 1. Let $M^{n}, n>4$, be a compact, locally irreducible manifold with nonnegative Ricci curvature. If $-\mathcal{W}+S /[(n-2)(n-1)] \geq 0$ then one of the following occurs:

(a) $M$ is covered by a compact symmetric space.

(b) The Betti numbers $\beta_{p}(M)=0$, for $1 \leq p \leq n-1$.

The key point of the proof of this result is to conclude that if $n>4$ then the restricted holonomy group of metrics with $\mathcal{Q}_{2} \geq 0$ and $-\mathcal{W}+S /[(n-$ $2)(n-1)] \geq 0$ is essentially $S O(n)$.

In the next result we assume a weaker condition for the Ricci curvature, namely, that the manifold $M$ has 2-nonnegative Ricci operator, that is to say the sum of the smallest 2 eigenvalues of the Ricci operator is nonnegative. We will consider such a condition on manifolds whose Weyl operator $\mathcal{W}$ commutes with Ric $\wedge I$, where Ric and $I$ denote the Ricci and the identity operators respectively. For such manifolds we have the following result:

Theorem 2. Let $M^{n}, n>4$, be a compact, locally irreducible manifold with 2-nonnegative Ricci operator. Let us suppose that $[\operatorname{Ric} \wedge I, \mathcal{W}]=0$ and $-\mathcal{W}+S /[(n-2)(n-1)] \geq 0$. Then one of following occurs:

(a) $M$ is covered by a compact symmetric space.

(b) The Betti numbers $\beta_{p}(M)=0$, for $2 \leq p \leq n-2$.

Observe that the Weyl operator of three important classes of manifolds commutes with Ric $\wedge I$ : Conformally flat, Einstein and manifolds with pure curvature tensor (see definition on page 439 of [4]). We also show that two other types of metrics satisfy the condition $[\operatorname{Ric} \wedge I, \mathcal{W}]=0$. They are $G$ manifolds of cohomogeneity one and Riemannian manifolds with harmonic curvature, non-parallel Ricci tensor and such that the operator Ric has less than three distinct eigenvalues. The last class of manifolds was studied by Derdzisnki in [7] and [8]. Such manifolds were the first examples of compact manifolds with harmonic curvature and non-parallel Ricci tensor and hence not Einstein. Among them we find, for $n>4$, examples that are not conformally flat either.

We also prove that locally reducible conformally flat manifolds with 2nonnegative Ricci operator in fact have nonnegative Ricci curvature. Using this fact, Theorem 1 above implies the corollary below, which generalizes Theorem 1 of [13].

Corollary 1. Let $M^{n}, n \geq 4$, be a compact conformally flat manifold with 2-nonnegative Ricci operator. Then either $M$ is flat or $\beta_{p}(M)=0$ for $2 \leq p \leq n-2$. Moreover if $\beta_{1}(M) \neq 0$ then $M$ is a quotient of $S^{n-1} \times \mathbf{R}$ or $\mathbf{R}^{n}$ by a group of fixed point free isometries in the standard metrics. 
We point out that for locally irreducible conformally flat manifolds, Theorem 2 above and Theorem 2 of [10] have the same conclusion, namely, that $\beta_{p}(M)=0$, for $2 \leq p \leq n-2$. This gives rise to a corollary with the same proof as Corollary 1 in [10]:

Corollary 2. Let $f: M^{n} \rightarrow \mathbf{R}^{n+p}, 2 \leq p \leq n / 2-1$, be an isometric immersion of a compact, orientable, locally irreducible conformally flat manifold $M$ with 2-nonnegative Ricci operator. Then $H_{i}(M ; \mathbf{Z})=0$ for $p \leq i \leq n-p$.

Another similarity between the topology of manifolds of 2-nonnegative Ricci operator and nonnegative isotropic curvature appears in the context of hypersurfaces of Euclidean spaces. For these, we prove the result below (compare with Theorem 1 of $[\mathbf{1 0}]$ ):

Theorem 3. Let $f: M^{n} \rightarrow \mathbf{R}^{n+1}, n \geq 4$, be an isometric immersion of a compact manifold $M$ with 2-nonnegative Ricci operator. Then the homology groups

$$
H_{i}(M ; \mathbf{Z})=0 \quad \text { for } \quad 2 \leq i \leq n-2
$$

and the fundamental group $\pi_{1}(M)$ is a free group on $\beta_{1}$ elements.

\section{Manifolds with nonnegative Weitzenböck operator.}

Let $M$ be a Riemannian manifold and Ric : $T_{x} M \rightarrow T_{x} M$ denote the Ricci operator given by

$$
\langle\operatorname{Ric}(X), Y\rangle=\operatorname{Ric}(X, Y) .
$$

In this paper we will use the same notation for a tangent vector $X$ and its dual form. With this in mind, we define the Weitzenböck operator $\mathcal{Q}_{2}$ : $\Lambda^{2}\left(T_{x} M\right) \rightarrow \Lambda^{2}\left(T_{x} M\right)$ as

$$
\begin{aligned}
\mathcal{Q}_{2}(X \wedge Y) & =(\operatorname{Ric} \wedge I)(X \wedge Y)-2 \mathcal{R}(X \wedge Y) \\
& =\operatorname{Ric}(X) \wedge Y+X \wedge \operatorname{Ric}(Y)-2 \mathcal{R}(X \wedge Y),
\end{aligned}
$$

where $\mathcal{R}$ is the curvature operator and $\Lambda^{2}\left(T_{x} M\right)$ denotes the space of 2 forms. This operator satisfies the well-known Weitzenböck formula, e.g., $\Delta \omega=-\operatorname{div} \nabla \omega+\mathcal{Q}_{2}(\omega)$, where $\Delta$ is the Laplace-Beltrami operator and $\nabla \omega$ the covariant derivative of $\omega$.

It is easy to see that $\mathcal{Q}_{2}$ is a self-adjoint operator, and therefore it makes sense to study it when it is nonnegative. The nonnegativity of the Weitzenböck operator has been used to study the second Betti number of compact manifolds. In this section we collect some results along this line.

Lemma 2.1. Let $M$ be a Riemannian manifold with nonnegative Weitzenböck operator. Then:

(a) If $e_{1}, e_{2}$ are orthonormal vectors, we have $\operatorname{Ric}\left(e_{1}, e_{1}\right)+\operatorname{Ric}\left(e_{2}, e_{2}\right)-$ $2 K_{12} \geq 0$, where $K_{12}$ is the sectional curvature of the plane spanned by $e_{1}$ and $e_{2}$. 
(b) The scalar curvature $S$ is nonnegative.

(c) If $S \equiv 0$ and $n>4$ then $M$ is flat.

(d) If $S \equiv 0$ and $n=4$ then $M$ is conformally flat.

Proof. Since $\mathcal{Q}_{2} \geq 0$, (a) comes straightforward from the definition of $\mathcal{Q}_{2}$.

Now we have that $\operatorname{Ric}\left(e_{1}, e_{1}\right)+\operatorname{Ric}\left(e_{j}, e_{j}\right)-2 K_{1 j} \geq 0$ for all unit vectors $e_{j}$ that are orthogonal to $e_{1}$. We obtain

$$
(n-1) \operatorname{Ric}\left(e_{1}, e_{1}\right)+\sum_{j \neq 1}\left[\operatorname{Ric}\left(e_{j}, e_{j}\right)-2 K_{1 j}\right]=(n-4) \operatorname{Ric}\left(e_{1}, e_{1}\right)+S \geq 0 .
$$

Therefore, if $n=4$ we have $S \geq 0$. If $n>4$ and the Ricci curvature is nonnegative then $S \geq 0$. If $\operatorname{Ric}\left(e_{1}, e_{1}\right)<0$ then $S>0$.

To prove (c), observe that Equation (2.2) also implies that if $S \equiv 0$ and $n>4$ then the Ricci curvature is nonnegative. If $S \equiv 0$, we conclude that $M$ is Ricci flat. This substituted in (a) implies that the sectional curvature $K \leq 0$ which gives $K=0$, again because $S \equiv 0$. The result in (d) is well-known (see for instance [12], Proposition 2.5 or [15], Proposition 2.5).

Proposition 2.3. Let $M^{n}, n \geq 4$, be a locally irreducible compact manifold with nonnegative Weitzenböck operator. Then:

(a) If $M$ is even dimensional and $\beta_{2}(M) \neq 0$ then $\beta_{2}(M)=1$ and $M$ is a simply connected Kähler manifold with positive first Chern class. Further, if $n=4$, then $M$ is biholomorphic to the complex projective space $\mathbf{C P}^{2}$.

(b) If $M$ is odd dimensional and $\beta_{2}(M) \neq 0$ then $M$ is covered by symmetric space of [compact type] and rank $>1$.

Proof. Since $M$ is compact, it follows from the nonnegativity of $\mathcal{Q}_{2}$ and the Weitzenböck formula that a harmonic 2-form $\omega$ is parallel.

If $M$ is even dimensional, the proof of Theorem 2.1(b) of [12] applies here, since it depends only on the fact that harmonic 2 -forms are parallel and $S \geq 0$ but not zero. Since $S=0$ implies that $M$ is flat and this contradicts the irreducibility of $M$ we conclude the first assertion of (a). The second part follows from Theorem 1 of [14].

If $M$ is odd dimensional, since we are supposing that $M$ is locally irreducible, then so is the restricted holonomy group $G$. Recall that in [2], Berger proved that if for some $x \in M, G$ acts irreducibly on $T_{x} M$, then either $M$ is locally symmetric or $G$ is one of the standard subgroups of $S O(n)$ :

$$
\begin{aligned}
& S O(n), U(m)(n=2 m), S p(m) \times S p(1)(n=4 m>4), \operatorname{Spin}(9)(n=16) \\
& S U(m)(n=2 m>2), S p(m)(n=4 m>4), G_{2}(n=7), \operatorname{Spin}(7)(n=8) .
\end{aligned}
$$

In the case that $M$ is locally symmetric, the universal cover $\widetilde{M}$ is an irreducible symmetric space. Since $\widetilde{M}$ is Einstein, if $S=0, M$ would be 
Ricci flat and then flat by Lemma 2.1. Therefore $S>0$ and $\widetilde{M}$ is compact. If $\operatorname{rank}(\widetilde{M})=1$, being odd-dimensional, it would be isometric to a sphere contradicting that $\beta_{2}(M) \neq 0$. Berger also proved that if $G$ is one of the possibilities listed on the second line above, $M$ is Ricci flat, which in this case implies that $M$ is flat. Note that in the other possibilities for $G, M$ is even dimensional, except if $G=S O(n)$. In this case, the existence of a parallel 2-form $\omega$ would give rise to a parallel and hence harmonic 2-form on $S^{n}$ by the holonomy principle, and this is a contradiction.

\section{A special condition on the Weyl tensor.}

We start this section proving a result for manifolds with nonnegative Weitzenböck operator and Weyl tensor satisfying a condition which generalizes conformal flatness. Before we state the result, we recall that the Weyl tensor induces an operator

$$
\mathcal{W}: \Lambda^{2}\left(T_{x} M\right) \rightarrow \Lambda^{2}\left(T_{x} M\right)
$$

given by

$$
\mathcal{W}(X \wedge Y)=\mathcal{R}(X \wedge Y)-\Gamma(X) \wedge Y-X \wedge \Gamma(Y)
$$

where $\Gamma: T_{x} M \rightarrow T_{x} M$ is defined by

$$
\Gamma(X)=\frac{1}{n-2}\left(\operatorname{Ric}(X)-\frac{S}{2(n-1)} X\right) .
$$

It is well-known that conformal flatness for manifolds of dimension $n \geq 4$ is equivalent to $\mathcal{W} \equiv 0$.

Lemma 3.1. Let $\mathcal{Q}_{2}, \mathcal{R}, \mathcal{W}$ denote the Weitzenböck, curvature and Weyl operator respectively. We have:

(a)

$$
\mathcal{Q}_{2}-(n-4) \mathcal{R}=\frac{S}{n-1}-(n-2) \mathcal{W} .
$$

(b) If $-\mathcal{W}+S /[(n-2)(n-1)] \geq 0$ and $\mathcal{Q}_{2}$ is a nonnegative operator $\left(\mathcal{Q}_{2} \geq 0\right)$ then

$$
\mathcal{Q}_{2}-2(p-2) \mathcal{R} \geq 0 \quad \text { whenever } \quad p \leq[n / 2] .
$$

Proof. Using the definition of $\mathcal{W}$ we obtain (a).

For (b), observe first that the assumptions imply that $\mathcal{Q}_{2}-(n-4) \mathcal{R}$ is a nonnegative operator. Now let $\mu$ be an eigenvalue of $\mathcal{Q}_{2}-2(p-2) \mathcal{R}$ with corresponding eigenvector $\phi$. If $\langle\mathcal{R}(\phi), \phi\rangle \leq 0$, then $\left\langle\left(\mathcal{Q}_{2}-2(p-\right.\right.$ $2) \mathcal{R})(\phi), \phi\rangle \geq 0$, since we are supposing that $\mathcal{Q}_{2} \geq 0$. If $\langle\mathcal{R}(\phi), \phi\rangle \geq 0$, we have for $p \leq[n / 2]$

$$
\left\langle\left(\mathcal{Q}_{2}-2(p-2) \mathcal{R}\right)(\phi), \phi\right\rangle \geq\left\langle\left(\mathcal{Q}_{2}-(n-4) \mathcal{R}\right)(\phi), \phi\right\rangle \geq 0 .
$$


Theorem 3.2. Let $M^{n}, n \geq 4$ be a locally irreducible compact manifold with nonnegative Weitzenböck operator and such that $-\mathcal{W}+S /[(n-2)(n-1)] \geq 0$. Then one of the following occurs:

(a) $M$ is locally symmetric and covered by a compact symmetric space.

(b) The Betti numbers $\beta_{p}(M)=0$ for $2 \leq p \leq n-2$.

(c) $M$ is 4-dimensional manifold biholomorphic to the complex projective space $\mathbf{C P}^{2}$.

Proof. Without loss of generality, we asume that $M$ is orientable. Let $\omega$ be a harmonic $p$-form. We use the Weitzenböck formula for $p$-forms (see [9])

$$
(\Delta \omega, \omega)=(\nabla \omega, \nabla \omega)+\int_{M} F(\omega) d V
$$

where $($,$) denotes the L^{2}$-product with respect to the Riemannian volume density $d V$ and

with

$$
F(\omega)=\frac{1}{(p-1) !}\left[A-\frac{p-1}{2} B\right]
$$

$$
\begin{aligned}
& A= \sum_{i_{3}, \ldots, i_{p}} \sum_{r, s, u, t} \omega\left(X_{s}, X_{r}, X_{i_{3}}, \ldots, X_{i_{p}}\right) \\
& \cdot \omega\left(X_{t}, X_{r}, X_{i_{3}}, \ldots, X_{i_{p}}\right)\left\langle R\left(X_{s}, X_{u}\right) X_{u}, X_{t}\right\rangle \\
& B=\sum_{i_{3}, \ldots, i_{p}} \sum_{r, s, u, t} \omega\left(X_{r}, X_{s}, X_{i_{3}}, \ldots, X_{i_{p}}\right) \\
& \cdot \omega\left(X_{t}, X_{u}, X_{i_{3}}, \ldots, X_{i_{p}}\right)\left\langle R\left(X_{r}, X_{s}\right) X_{u}, X_{t}\right\rangle .
\end{aligned}
$$

Notice that $F(\omega)$ can be written as

$$
F(\omega)=\frac{1}{(p-1) !} \sum_{i_{3}, \ldots, i_{p}}\left\langle\left(\mathcal{Q}_{2}-2(p-2) \mathcal{R}\right)\left(\phi_{i_{3}, \ldots, i_{p}}\right), \phi_{i_{3}, \ldots, i_{p}}\right\rangle
$$

where $\phi_{i_{3}, \ldots, i_{p}}$ is a 2 -form obtained by fixing $X_{i_{3}}, \ldots, X_{i_{p}}$ and defining

$$
\phi_{i_{3}, \ldots, i_{p}}(u, v)=\omega\left(u, v, X_{i_{3}}, \ldots, X_{i_{p}}\right) .
$$

Therefore, $\mathcal{Q}_{2}-2(p-2) \mathcal{R} \geq 0$ implies $F(\omega) \geq 0$.

Proceeding as the proof of Lemma 3.1(b), we conclude that $\left(\mathcal{Q}_{2}-2(p-\right.$ $2) \mathcal{R}) \geq 0$ for $2 \leq p \leq[n / 2]$ and hence for $p$ in this range, a harmonic $p$-form is parallel. Again, we study each possibility for the restricted holonomy group $G$ and use the holonomy principle.

If $M$ is locally symmetric, being locally irreducible, $\widetilde{M}$ is an irreducible symmetric space and therefore an Einstein space. Since it cannot be Ricci flat, it has positive Ricci curvature and hence compact.

The fact that $M$ cannot be Ricci flat leaves us with the following possibilities:

$$
S O(n), U(m)(n=2 m), S p(m) \times S p(1)(n=4 m>4), \operatorname{Spin}(9)(n=16) .
$$


In the last case, a result in [5] implies that $M$ is locally symmetric, and we repeat the previous argument.

Recall that if $\beta_{2}(M) \neq 0$, and $M$ is even dimensional then $M$ is a Kähler manifold and then there exists a parallel form $\omega$ for which $\mathcal{Q}_{2}(\omega)=0$. Moreover we can find orthonomal vectors $e_{1}, \ldots, e_{m}, n=2 m$, such that

$$
\omega=J\left(e_{1}\right) \wedge e_{1}+\cdots+J\left(e_{m}\right) \wedge e_{m},
$$

where $J$ denotes the complex structure on $M$. Using the fact that $\mathcal{Q}_{2}(\omega)=0$, from the definition of $\mathcal{Q}_{2}$ we get

$$
\begin{aligned}
0= & \operatorname{Ric}\left(e_{1}, e_{1}\right)+\operatorname{Ric}\left(J\left(e_{1}\right), J\left(e_{1}\right)\right)+\cdots+\operatorname{Ric}\left(e_{m}, e_{m}\right) \\
& +\operatorname{Ric}\left(J\left(e_{m}\right), J\left(e_{m}\right)\right)-2\langle\mathcal{R}(\omega), \omega\rangle,
\end{aligned}
$$

which gives

$$
\langle\mathcal{R}(\omega), \omega\rangle=\frac{S}{2}
$$

yielding

$$
\langle\mathcal{W}(\omega), \omega\rangle=\frac{(n-2) S}{2(n-1)} .
$$

On the other hand, let $\left\{\phi_{i}\right\}$ denote an orthonormal basis which diagonalizes $\mathcal{W}$ with corresponding eigenvalues $\nu_{i}$. We then write $\omega=\sum_{i} a_{i} \phi_{i}$, and then

$$
\langle\mathcal{W}(\omega), \omega\rangle=\frac{(n-2) S}{2(n-1)}=\sum_{i} a_{i}^{2} \nu_{i}
$$

Let us suppose that the eigenvalues $\nu_{i}$ 's are increasing and let $i_{0}$ denote the index such that $\nu_{i} \geq 0$, for $i \geq i_{0}$. Therefore, from our assumption on the eigenvalues of the Weyl operator, we get

$$
\frac{(n-2) S}{2(n-1)} \leq \sum_{i \geq i_{0}} a_{i}^{2} \frac{S}{(n-1)(n-2)} \leq \frac{S}{(n-1)(n-2)} \frac{n}{2} .
$$

But the above implies either $(n-2)^{2} \leq n$, which is clearly a contradiction for $n>4$, or $S=0$. But $S=0$ contradicts the irreducibility of $M$, since it implies that $M$ is flat. Therefore, if $n>4, \beta_{2}(M)=0$ and hence the holonomy $G$ cannot be $U(m)$. If $n=4$, we obtain that (c) follows from Proposition 2.3.

If $G=S p(m) \times S p(1), M$ is Einstein (see [3]) and hence has positive Ricci curvature. Furthermore, $M$ is a quaternionic Kähler manifold which implies the existence of a parallel 4-form (V.Y. Kraines, see [4] p. 419), which we denote by $\omega$, and then $F(\omega)=0$. From the equation

$$
F(\omega)=\frac{1}{(3) !} \sum_{i_{3}, i_{4}}\left\langle\left(\mathcal{Q}_{2}-4 \mathcal{R}\right)\left(\phi_{i_{3}, i_{4}}\right), \phi_{i_{3}, i_{4}}\right\rangle,
$$


and the fact that $\left(\mathcal{Q}_{2}-4 \mathcal{R}\right)$ is a nonnegative operator, we obtain

$$
\left\langle\left(\mathcal{Q}_{2}-4 \mathcal{R}\right)\left(\phi_{i_{3}, i_{4}}\right),\left(\phi_{i_{3}, i_{4}}\right)\right\rangle=0,
$$

for all 2 -forms of type $\phi_{i_{3}, i_{4}}$. On the other hand, we have $\left\langle\left(\mathcal{Q}_{2}-(n-\right.\right.$ $\left.4) \mathcal{R})\left(\phi_{i_{3}, i_{4}}\right),\left(\phi_{i_{3}, i_{4}}\right)\right\rangle \geq 0$, and using (3.3) we obtain

$$
(8-n)\left\langle\mathcal{R}\left(\phi_{i_{3}, i_{4}}\right), \phi_{i_{3}, i_{4}}\right\rangle \geq 0 .
$$

If $n>8$, the above implies that $\left\langle\mathcal{Q}_{2}\left(\phi_{i_{3}, i_{4}}\right), \phi_{i_{3}, i_{4}}\right\rangle=4\left\langle\mathcal{R}\left(\phi_{i_{3}, i_{4}}\right), \phi_{i_{3}, i_{4}}\right\rangle \leq 0$. Since $\mathcal{Q}_{2} \geq 0$, we then have that

$$
\left\langle\mathcal{Q}_{2}\left(\phi_{i_{3}, i_{4}}\right), \phi_{i_{3}, i_{4}}\right\rangle=\left\langle\mathcal{R}\left(\phi_{i_{3}, i_{4}}\right), \phi_{i_{3}, i_{4}}\right\rangle=0 .
$$

But $M$ is Einstein and hence $\mathcal{Q}_{2}=2(S / n)-2 \mathcal{R}$. Therefore, the equation above gives

$$
\frac{2 S\left\|\phi_{i_{3}, i_{4}}\right\|^{2}}{n}=0
$$

implying $S=0$, which is the desired contradiction.

If $n=8,(3.3)$ substituted in Lemma 3.1(a) immediately implies

$$
\left\langle\mathcal{W}\left(\phi_{i_{3}, i_{4}}\right), \phi_{i_{3}, i_{4}}\right\rangle=\frac{S\left\|\phi_{i_{3}, i_{4}}\right\|^{2}}{42}
$$

and we claim that $\phi_{i_{3}, i_{4}}$ is an eigenvector of $\mathcal{W}$. In fact, if not, we consider again an orthonormal basis $\left\{\phi_{i}\right\}$ which diagonalizes $\mathcal{W}$, and let $i_{0}$ denote the index such that $\nu_{i} \geq 0$, for $i \geq i_{0}$. We would have

$$
\left\langle\mathcal{W}\left(\phi_{i_{3}, i_{4}}\right), \phi_{i_{3}, i_{4}}\right\rangle<\sum_{i \geq i_{0}} a_{i}^{2} \frac{S}{42}<\frac{S\left\|\phi_{i_{3}, i_{4}}\right\|^{2}}{42} .
$$

Since $M$ is Einstein, an eigenvector of $\mathcal{W}$ is also an eigenvector $\mathcal{R}$, and then we obtain that

$$
\mathcal{R}\left(\phi_{i_{3}, i_{4}}\right)=\frac{S}{24} \phi_{i_{3}, i_{4}} .
$$

We will show now that one can obtain a basis of $\Lambda^{2}\left(T_{x} M\right)$ whose elements are 2 -forms of type $\phi_{i_{3}, i_{4}}$. This implies $\mathcal{R}=(S / 24) I$, that is, $M$ is a manifold of constant curvature contradicting that its restricted holonomy group $G$ is $S p(2) \times S p(1)$. For that, let $I, J, K$ denote the almost complex structures of $M$ which satisfy the relations $I J=-J I$, and $K=I J$. Let $\left\{e_{1}, \ldots, e_{8}\right\}$ be an orthonormal basis with the property

$$
\begin{array}{lll}
e_{2}=I\left(e_{1}\right), & e_{3}=J\left(e_{1}\right), & e_{4}=K\left(e_{1}\right) \\
e_{6}=I\left(e_{5}\right), & e_{7}=J\left(e_{5}\right), & e_{8}=K\left(e_{5}\right) .
\end{array}
$$

The form $\omega$ is given by

$$
\omega=\alpha \wedge \alpha+\beta \wedge \beta+\gamma \wedge \gamma
$$

where

$$
\alpha(X, Y)=\langle I(X), Y\rangle \quad \beta(X, Y)=\langle J(X), Y\rangle \quad \gamma(X, Y)=\langle K(X), Y\rangle .
$$


Therefore $\alpha, \beta$ and $\gamma$ are written as

$$
\begin{aligned}
& \alpha=e_{1} \wedge e_{2}+e_{3} \wedge e_{4}+e_{5} \wedge e_{6}+e_{7} \wedge e_{8} \\
& \beta=e_{1} \wedge e_{3}-e_{2} \wedge e_{4}+e_{5} \wedge e_{7}-e_{6} \wedge e_{8} \\
& \gamma=e_{1} \wedge e_{4}+e_{2} \wedge e_{3}+e_{5} \wedge e_{8}+e_{6} \wedge e_{7},
\end{aligned}
$$

and then

$$
\begin{aligned}
\omega= & 6 e_{1} \wedge e_{2} \wedge e_{3} \wedge e_{4}+2 e_{1} \wedge e_{2} \wedge e_{5} \wedge e_{6} \\
& +2 e_{1} \wedge e_{2} \wedge e_{7} \wedge e_{8}+2 e_{1} \wedge e_{3} \wedge e_{5} \wedge e_{7}-2 e_{1} \wedge e_{3} \wedge e_{6} \wedge e_{8} \\
& +2 e_{1} \wedge e_{4} \wedge e_{5} \wedge e_{8}+2 e_{1} \wedge e_{4} \wedge e_{6} \wedge e_{7}+2 e_{2} \wedge e_{3} \wedge e_{5} \wedge e_{8} \\
& +2 e_{2} \wedge e_{3} \wedge e_{6} \wedge e_{7}-2 e_{2} \wedge e_{4} \wedge e_{5} \wedge e_{7}+2 e_{2} \wedge e_{4} \wedge e_{6} \wedge e_{8} \\
& +2 e_{3} \wedge e_{4} \wedge e_{5} \wedge e_{6}+2 e_{3} \wedge e_{4} \wedge e_{7} \wedge e_{8}+6 e_{5} \wedge e_{6} \wedge e_{7} \wedge e_{8} .
\end{aligned}
$$

As before, let us consider the 2 -form $\phi_{i, j}(u, v)=\omega\left(u, v, e_{i}, e_{j}\right)$. From the expression of $\omega$ it is straightforward to conclude that $\left\{\phi_{i, j}, i<j\right\}$ is a basis of $\wedge^{2}\left(T_{x} M\right)$. Since $G \neq U(m), S p(m) \times S p(1)$, if $n>4$ then the only possibility for $G$ is $S O(n)$. The holonomy principle implies that $\beta_{p}(M)=0$ for $2 \leq p \leq[n / 2]$ and we conclude $\beta_{p}(M)=0$ for $2 \leq p \leq n-2$ by duality.

Now we use Theorem 3.2 to prove Theorem 1 stated in the introduction. Proof of Theorem 1 . We show that the hypotheses imply $\mathcal{Q}_{2} \geq 0$. In fact, let $\omega$ be a unit eigenvector of $\mathcal{Q}_{2}$. There exist an orthonormal set $\left\{e_{1}, \ldots, e_{2 m}\right\}$ of $T_{x} M$ and numbers $a_{1}, \ldots, a_{m}$ such that

$$
\omega=a_{1} e_{1} \wedge e_{2}+\cdots+a_{m} e_{2 m-1} \wedge e_{2 m} .
$$

From the definition of $\mathcal{Q}_{2}$ we obtain

$$
\begin{aligned}
\left\langle\mathcal{Q}_{2}(\omega), \omega\right\rangle= & \sum_{i=1}^{m} a_{i}^{2}\left(\operatorname{Ric}\left(e_{2 i-1}, e_{2 i-1}\right)+\operatorname{Ric}\left(e_{2 i}, e_{2 i}\right)\right)-2\langle\mathcal{R}(\omega), \omega\rangle \\
= & \frac{n-4}{n-2} \sum_{i=1}^{m} a_{i}^{2}\left(\operatorname{Ric}\left(e_{2 i-1}, e_{2 i-1}\right)+\operatorname{Ric}\left(e_{2 i}, e_{2 i}\right)\right) \\
& -2\left[\langle\mathcal{W}(\omega), \omega\rangle-\frac{S}{(n-2)(n-1)}\right] \geq 0 .
\end{aligned}
$$

Now, Theorem 3.2 implies (a) or $\beta_{p}(M)=0$ for $2 \leq p \leq n-2$. Since we are also assuming that all Ricci curvatures are nonnegative, we apply the well-known generalization of Bochner's theorem, namely, that either $M$ is covered by a compact symmetric space or it is Ricci flat or $\beta_{1}(M)=0$. Since our hypotheses imply $\mathcal{Q}_{2} \geq 0, \mathrm{M}$ cannot be Ricci flat and this finishes the proof of the theorem. 
Next we want to examine another condition on the Weyl operator that also generalizes $\mathcal{W}=0$. Such a condition is

$$
[\operatorname{Ric} \wedge I, \mathcal{W}]=0 .
$$

This condition is satisfied by several important classes of Riemannian manifolds. Among them, we easily find the Einstein manifolds. In this section we show other classes of manifolds whose Weyl operator commutes with $\operatorname{Ric} \wedge I$.

Recall that the curvature operator $\mathcal{R}$ is said to be pure if there exists an orthonormal basis $\left\{e_{1}, \ldots, e_{n}\right\}$ of the tangent space such that the basis of 2 -forms $\left\{e_{i} \wedge e_{j}\right\}_{i<j}$ diagonalizes $\mathcal{R}$. We call the basis $\left\{e_{1}, \ldots, e_{n}\right\}$ an $\mathcal{R}$-basis.

Notice that the Weyl tensor of a manifold with pure curvature operator satisfies $[$ Ric $\wedge I, \mathcal{W}]=0$. This class of manifolds also includes hypersurfaces of Euclidean spaces, and more generally, manifolds which admit isometric immersions into a space of constant curvature with flat normal bundle. To see this, just use the Ricci equation which implies that there is an orthonormal basis that diagonalizes simultaneously all the Weingarten operators; then from the Gauss equation one obtains the $\mathcal{R}$-basis. The technical condition of the next lemma will appear naturally in two other classes of manifolds.

Lemma 3.4. Let $M$ be a Riemannian manifold such that for every point $x \in M$, the Ricci operator $\operatorname{Ric}_{x}$ has an eigenvalue $\lambda(x)$ of constant multipicity $n-1$. Suppose that the eigenspaces $E_{\lambda}$ corresponding to $\lambda$ form an integrable distribution. If their leaves are totally umbilic submanifolds and have constant mean curvature then $[\operatorname{Ric} \wedge I, \mathcal{W}]=0$.

Proof. Let $\left\{e_{1}, \ldots, e_{n}\right\}$ be an orthonormal basis such that $\operatorname{Ric}\left(e_{1}\right)=\mu e_{1}$ and Ric $\left(e_{i}\right)=\lambda e_{i}$, for $i \geq 2$. We show first that

$$
\left\langle\mathcal{R}\left(e_{1} \wedge e_{k}\right), e_{i} \wedge e_{j}\right\rangle=0, \quad i, j, k \geq 2 .
$$

For that, let $\Sigma$ denote a maximal leaf of $E_{\lambda}$. Let $A$ denote the shape operator of the inclusion $i: \Sigma \rightarrow M$ with only an eigenvalue of multiplicity $n-1$ denoted by $a$. Since $a$ is constant, it is straightforward to verify that $A$ satifies the Codazzi equation

$$
\left\langle\left(\bar{\nabla}_{e_{i}} A\right)\left(e_{j}\right), e_{k}\right\rangle=\left\langle\left(\bar{\nabla}_{e_{j}} A\right)\left(e_{i}\right), e_{k}\right\rangle, \quad \forall i, j, k \geq 2,
$$

where $\bar{\nabla}$ is the induced connection on $\Sigma$. This fact implies $\left\langle\mathcal{R}\left(e_{i} \wedge e_{j}\right), e_{1} \wedge\right.$ $\left.e_{k}\right\rangle=0$. Then, we have that

$$
\mathcal{W}\left(e_{1} \wedge e_{k}\right)=\mathcal{R}\left(e_{1} \wedge e_{k}\right)-\frac{\mu}{n-1} e_{1} \wedge e_{k}
$$

lies in the space $V=\operatorname{span}\left\{e_{1} \wedge e_{k}, k \geq 2\right\}$. Since Ric $\wedge I$ restricted to $V$ is a multiple of the identity, we have that $\mathcal{W}$ and Ric $\wedge I$ commute on $V$. We 
also have, for $i, j \geq 2$,

$$
\mathcal{W}\left(e_{i} \wedge e_{j}\right)=\mathcal{R}\left(e_{i} \wedge e_{j}\right)-\frac{(n-1) \lambda-\mu}{(n-1)(n-2)} e_{i} \wedge e_{j},
$$

and then $\mathcal{W}\left(e_{i} \wedge e_{j}\right) \in U=\operatorname{span}\left\{e_{i} \wedge e_{j}, i, j \geq 2, i<j\right\}$ implying that $[\operatorname{Ric} \wedge I, \mathcal{W}]=0$ on $U$.

Definition 3.5. A Riemannian $G$-manifold is said to be of cohomogeneity one if the group $G$ acts effectively and isometrically with principal orbits of codimension one.

Proposition 3.6. Let $M$ be a cohomogeneity one $G$-manifold such that its principal orbits are isotropy-irreducible homogeneous spaces (see [4, p. 187]). Then the set of regular points $M_{\mathrm{reg}}$ of $M$ satisfies the conditions of Lemma 3.4. It follows that $[\mathrm{Ric} \wedge I, \mathcal{W}]=0$ for all points of $M$.

Proof. Since $\Sigma$ is an isotropy-irreducible homogeneous space, the immersion $i: \Sigma \rightarrow M$ is totally umbilic. Further, a $G$ invariant metric defined on an isotropy-irreducible homogeneous space is Einstein. From this and the fact that the immersion of the orbit $\Sigma$ into $M$ is totally umbilic, we obtain that

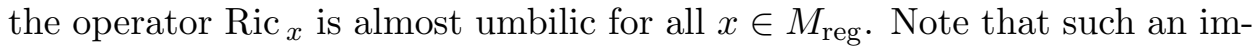
mersion has constant mean curvature, by the homogeneity of $\Sigma$. Therefore, from the Lemma above we get that $[\operatorname{Ric} \wedge I, \mathcal{W}]=0$ on 2 -forms defined on $\Lambda^{2}\left(T_{x} M\right)$ for $x \in M_{\text {reg }}$. Since $M_{\text {reg }}$ is dense in $M$, we have the result.

Proposition 3.7. Let $M$ be a Riemannian manifold with harmonic curvature and non-parallel Ricci tensor. If Ric has less than three distinct eigenvalues at any point of $M$ then $M$ satisfies the conditions of Lemma 3.4 and hence $[$ Ric $\wedge I, \mathcal{W}]=0$ for all points of $M$.

Proof. The proof that $M$ satisfies the conditions of Lemma 3.4 is Lemma 3 of $[7]$.

Lemma 3.8. Let $M$ be Riemannian manifold with the property that [Ric $\wedge$ $I, \mathcal{W}]=0$. Let $\left\{e_{1}, \ldots, e_{n}\right\}$ be an orthonormal basis of eigenvectors of Ric with corresponding eigenvalues $\mu_{i}$. Then:

(a) $\mathcal{R}\left(e_{i} \wedge e_{j}\right)$ and $F\left(e_{i} \wedge e_{j}\right)$ are eigenvectors of Ric $\wedge I$ with corresponding eigenvalue $\mu_{i}+\mu_{j}$.

(b) Let $E_{\mu_{i}}$ denote the eigenspace of $\mu_{i}$. If $\left\{e_{1}, \ldots, e_{k}\right\}$ is a basis of $E_{\mu_{i}}$ and $\left\{e_{k+1}, \ldots, e_{m}\right\}$ a basis of $E_{\mu_{j}}$ then the space $\operatorname{span}\left\{e_{r} \wedge e_{s}, r=\right.$ $1, \ldots, k, s=k+1, \ldots, m\}$ is invariant by $\mathcal{R}$ and $\mathcal{Q}_{2}$.

Proof. Since the condition $[\operatorname{Ric} \wedge I, \mathcal{W}]=0$ implies that Ric $\wedge I$ commutes with $\mathcal{R}$ and $\mathcal{Q}_{2}$ and $\mu_{i}+\mu_{j}$ is an eigenvalue of Ric $\wedge I$ with corresponding eigenvector $e_{i} \wedge e_{j}$, we have Part (a), which immediately implies (b). 


\section{Manifolds with 2-nonnegative Ricci operator.}

It has been shown by H. Chen ([6]) that, from the topological point of view, compact manifolds with 2-nonnegative curvature operator are the same as the ones with nonnegative curvature operator. In this section we want to investigate to what extent the topology of manifolds with nonnegative Ricci curvature and 2-nonnegative Ricci operator can be compared.

Definition 4.1. The Ric operator is said to be 2-nonnegative (respectively, positive) if the sum of the first 2 eigenvalues is nonnegative (respectively, positive).

Proposition 4.2. Let $M^{n}$ be a locally reducible Riemannian manifold with 2-nonnegative Ricci operator. If $M$ does not have nonnegative Ricci curvature then the universal cover $\widetilde{M}$ is isometric $N_{1} \times \cdots \times N_{m}$ where each $N_{i}$ is irreducible and non-flat and one the $N_{i}$ 's is at least 3-dimensional and has 2-nonnegative Ricci operator and all other factors have nonnegative Ricci curvature.

Proof. The universal covering $\widetilde{M}$ is isometric to $\mathbf{R}^{k} \times N_{1} \times \cdots \times N_{m}$ by the decomposition theorem of de Rham. If $k \geq 1$ then $\operatorname{Ric}(X)=0$ for all $X$ that is tangent to $\mathbf{R}^{k}$ and then $M$ has nonnegative Ricci curvature. If $k=0$, and one of the $N_{i}$ 's is 2-dimensional, its curvature must be nonnegative, otherwise Ric would have 2 negative eigenvalues. Therefore, if Ric has a negative eigenvalue, one of the $N_{i}$ 's has dimension at least 3 . The remanining statements now are obvious.

Corollary 4.3. Let $M^{n}$ be a locally reducible conformally flat manifold with 2-nonnegative Ricci operator. Then $M$ has nonnegative Ricci curvature.

Proof. It follows from Proposition 4.2 that the only case to be studied here is $\widetilde{M}=N_{1} \times \cdots \times N_{m}$ where a factor $N_{i_{0}}$ has dimension $k \geq 3,2$ nonnegative Ricci operator, and all other factors are at least 2-dimensional. Let $\left\{e_{1}, \ldots, e_{k}\right\}$ be a basis of vectors tangent to $N_{i_{0}}$ and $e_{r}, e_{s}$ orthonormal vectors tangent to $N_{j}, j \neq i_{0}$. Since $M$ is conformally flat we have that

$$
K_{12}+K_{34}=K_{13}+K_{24},
$$

whenever $e_{1}, e_{2}, e_{3}, e_{4}$ are orthonormal vectors. Using this relation we get

$$
K_{i j}+K_{r s}=K_{i r}+K_{j s}=0, \quad \forall i, j=1, \ldots k, i \neq j .
$$

This implies that $N_{i_{0}}$ has constant curvature and hence positive Ricci curvature.

Now we are ready to prove Theorem 2 .

Proof of Theorem 2. Again we show that the hypotheses of the theorem imply that the Weitzenböck operator $\mathcal{Q}_{2}$ is nonnegative and the result will follow from Theorem 3.2. 
Let $\omega$ be a unit eigenvector of $\mathcal{Q}_{2}$. From Part (b) of Lemma 3.8 (and with same notation) we get that $\omega \in \operatorname{span}\left\{e_{r} \wedge e_{s}, r=1, \ldots, k, s=k+1, \ldots, m\right\}$, for some $i, j$. Since we have

$$
\begin{gathered}
\mathcal{R}(\omega)=\mathcal{W}(\omega)+\left(\frac{\mu_{i}+\mu_{j}}{n-2}\right) \omega-\frac{S}{(n-1)(n-2)} \omega \\
\mathcal{Q}_{2}(\omega)=\left(\mu_{i}+\mu_{j}\right) \omega-2 \mathcal{R}(\omega),
\end{gathered}
$$

we obtain

$$
\left\langle\mathcal{Q}_{2}(\omega), \omega\right\rangle=\frac{(n-4)\left(\mu_{i}+\mu_{j}\right)}{n-2}-2\left[\langle\mathcal{W}(\omega), \omega\rangle-\frac{S}{(n-1)(n-2)}\right] \geq 0 .
$$

Combining the results of Corollary 4.3, Theorem 2 and Theorem of [13] we obtain Corollary 1 stated in the introduction.

Now we use Theorem 2 to conclude the following results for the manifolds studied in the last section.

Theorem 4.4. Let $M^{n}, n \geq 5$, be a compact, locally irreducible cohomogeneity one $G$-manifold such that its principal orbits are isotropy-irreducible homogeneous spaces. If $M$ has 2-nonnegative Ricci operator and $-\mathcal{W}+$ $S /((n-1)(n-2)) \geq 0$ then $\beta_{i}(M)=0$, for $2 \leq i \leq n-2$.

Proof. Observe first that combining Proposition 3.6 and Theorem 2 we get either $\beta_{i}(M)=0$, for $2 \leq i \leq n-2$ or $\widetilde{M}$ is a compact symmetric space, and in particular a homogeneous space. A theorem of Podestà states (see [16]) that a compact homogeneous space that is also a cohomogeneity one manifold with isotropy-irreducible principal orbits is isometric to the sphere or to the real projective space which implies again that $\beta_{i}(M)=0$, for $2 \leq i \leq n-2$.

Theorem 4.4 above generalizes in some sense a result of Podestà in [17], which states that a compact $G$-cohomogeneity one manifold of positive Ricci curvature and isotropy-irreducible principal orbits is covered by a manifold conformally difeomorphic to a sphere.

For manifolds with harmonic curvature we obtain the following result.

Theorem 4.5. Let $M^{n}, n \geq 5$, be a compact locally irreducible Riemannian manifold with harmonic curvature and non-parallel Ricci tensor. Let us suppose that Ric has less than three distinct eigenvalues at any point of $M$ and the eigenvalues of the Weyl operator satisfy $-\mathcal{W}+S /((n-1)(n-2)) \geq 0$. If $M$ has 2-nonnegative Ricci operator then $\beta_{i}(M)=0$, for $2 \leq i \leq n-2$.

Proof. It is immediate from Proposition 3.7 and Theorem 2, since we are assuming that the Ricci tensor is non-parallel and hence $M$ cannot be locally symmetric. 


\section{Hypersurfaces of 2-nonnegative Ricci operator.}

It is well-known that on hypersurfaces of Euclidean spaces, nonnegative Ricci curvature implies the nonnegativity of the sectional curvatures. The next result shows that compact hypersurfaces with 2-nonnegative Ricci operator and compact hypersurfaces with nonnegative isotropic curvatures are topologically the same. This is the content of Theorem 3 stated in the introduction that we now prove.

Proof of Theorem 3. Let $\xi$ be a unit vector such that $\pm \xi$ are regular values of the Gauss map $\Phi: M^{n} \rightarrow S^{n} \subseteq \mathbf{R}^{n+1}$. Then the height function $h_{\xi}$ : $M \rightarrow \mathbf{R}$ given by $h_{\xi}(x)=\langle f(x), \xi\rangle$ is a Morse fuction with critical points $\Phi^{-1}( \pm \xi)$. At such points the Hessian of $h_{\xi}$ is given, up to a sign, by the Weingarten operator $A_{\xi}$. Let $\left\{e_{1}, \ldots, e_{n}\right\}$ be an orthonormal basis of $T_{x} M$ that diagonalizes $A_{\xi}$, say, $A_{\xi} e_{i}=\lambda_{i} e_{i}$. By the Gauss equation $K_{i j}=\lambda_{i} \lambda_{j}$ and since the critical points are nondegenerate, we have that $\lambda_{i} \neq 0$ for $i=1, \ldots, n$. As before, we denote the eigenvalues of the Ricci operator by $\mu_{i}$. If all eigenvalues of the Ricci operator are nonnegative, then the sectional curvatures $K_{i j} \geq 0$ and all eigenvalues of $A_{\xi}$ have the same sign.

Suppose $\mu_{1}<0$. We claim that in this case $n-1$ eigenvalues of $A_{\xi}$ have the same sign.

If $\lambda_{1}<0$, we reorder the $\lambda_{i}$ 's for $i \geq 2$, such that $\lambda_{2} \leq \cdots \leq \lambda_{n}$. Thus

$$
\mu_{1}=\lambda_{1}\left(\lambda_{2}+\lambda_{3}+\cdots+\lambda_{n}\right)<0 \Rightarrow \lambda_{2}+\lambda_{3}+\cdots+\lambda_{n}>0 .
$$

Therefore $\lambda_{n}>0$. Now we suppose that $\lambda_{2}<0$ and this will give a contradiction. Indeed, since our hypothesis implies $\mu_{2} \geq 0$ we have

$$
\mu_{2}=\lambda_{2}\left(\lambda_{1}+\lambda_{3}+\cdots+\lambda_{n}\right)>0 \Rightarrow \lambda_{1}+\lambda_{3}+\cdots+\lambda_{n}<0,
$$

which in turn implies

$$
\lambda_{3}+\cdots+\lambda_{n}<-\lambda_{1} .
$$

Since $\mu_{n} \geq 0$ and $\lambda_{n}>0$ we also have

$\mu_{n}=\lambda_{2} \lambda_{n}+\lambda_{n}\left(\lambda_{1}+\lambda_{3}+\cdots+\lambda_{n-1}\right)>0 \Rightarrow \lambda_{1}+\lambda_{3}+\cdots+\lambda_{n-1}>0$, yielding

$$
\lambda_{3}+\cdots+\lambda_{n-1}>-\lambda_{1} .
$$

From (5.1) and (5.2) we get $-\lambda_{1}+\lambda_{n}<-\lambda_{1}$ implying that $\lambda_{n}<0$ and this is a contradiction.

If $\lambda_{1}>0$ we then have that $\lambda_{2}+\lambda_{3}+\cdots+\lambda_{n}<0$. Since $\mu_{1}<0$ not all $\lambda_{i}$ 's have the same sign, otherwise all sectional curvatures would be positive. Let us the suppose that $\lambda_{2}<0$, after we have reordered such that $\lambda_{2} \leq \cdots \leq \lambda_{n}$. If $\lambda_{i}<0$ for $i \geq 3$ we have the claim. If not, then $\lambda_{n}>0$. Again we obtain (5.1) and (5.2) which gives the desired contradiction. 
Therefore we conclude that for each regular point, all but at most one of the $\lambda_{i}^{\prime} s$ have the same sign and hence the index of a critical point of $h_{\xi}$ has to be $0,1, n-1$ or $n$. By the standard Morse Theory, $M$ has the homotopy type of a $C W$-complex, with no cells of dimension $i$ for $2 \leq i \leq n-2$. Therefore the homology group $H_{i}(M ; \mathbf{Z})=0$ for $2 \leq i \leq n-2$. Moreover, since there are no 2 -cells $(n \geq 4)$, we conclude by the cellular aproximation theorem that the inclusion of the 1-skeleton $M^{(1)} \hookrightarrow M$ induces an isomorphism between the fundamental groups. Therefore the fundamental group $\pi_{1}(M)$ is a free group on $\beta_{1}$ elements and $H_{1}(M ; \mathbf{Z})$ is a free abelian group with the same number of generators.

Acknowledgements. The results of this article are extensions of some results in the first author's Ph.D. dissertation, which was directed by the second author. The second author wants to thank the Department of Mathematics of UNICAMP in Campinas, Brasil, for their hospitality and both authors would like to thank Professor Francesco Mercuri for helpful and valuable discussions.

\section{References}

[1] A.C. Asperti, F. Mercuri and M.H. Noronha, Cohomogeneity one manifolds and hypersurfaces of revolution, Bolletino U.M.I, 11 (1997), 199-215, MR 98c:53061, Zbl 0882.53006.

[2] M. Berger, Sur les groupes d'holonomie des variétés a connexion affine et des variétés riemannienes, Bull. Soc. Math. France, 83 (1955), 279-310, MR 18,149a, Zbl 0068.36002.

[3] Sur les groupes d'holonomie homogenes des variétés riemannienes, C.R. Acad. Sci. Paris serie A, 262 (1966), 1316.

[4] A. Besse, Einstein Manifolds, Ergeb. Math. Grenzgeb (3), 10, Springer-Verlag, Berlin, 1987, MR 88f:53087, Zbl 0613.53001.

[5] R.B. Brown and A. Gray, Riemannian manifolds with holonomy group immersed Spin (9), Differential Geometry (in honor of K. Yano), Kinokuniya, Tokyo, (1972), 41-59, MR 48 \#7159, Zbl 0245.53020.

[6] H. Chen, Manifolds with 2-nonnegative curvature operator, Proceed. of Symposia in Pure Math., 54 (1993), 129-133, MR 94f:53063, Zbl 0796.53040.

[7] A. Derdzinski, Classification of certain compact Riemannian manifolds with harmonic curvature and non-parallel Ricci tensor, Math. Z., 172 (1980), 273-280, MR 82e:53053, Zbl 0453.53037.

[8] _ On compact Riemannian manifolds with harmonic curvature, Math. Ann., 259 (1982), 145-152, MR 83i:53070, Zbl 0489.53042.

[9] S. Gallot and D. Meyer, Opérateur de courbure et Laplacien des formes différentelles d'une variété Riemanniene, J. Math. Pures et Appl., 54 (1975), 285-304, MR 56 \#13128, Zbl 0316.53036. 
[10] F. Mercuri and M.H. Noronha, Low codimensional submanifolds of euclidean space with nonnegative isotropic curvature, Trans. A.M.S., 348 (1996), 2711-2724, MR 96j:53049, Zbl 0862.53003.

[11] M. Micallef and J.D. Moore, Minimal two-spheres and the topology of manifolds with positive curvature on totally isotropic two-planes, Ann. of Math., 127 (1988), 199-227, MR 89e:53088, Zbl 0661.53027.

[12] M. Micallef and M.Y. Wang, Metrics with nonnegative isotropic curvature, Duke Math. J., 72 (1993), 649-672, MR 94k:53052, Zbl 0804.53058.

[13] M.H. Noronha, Some compact conformally flat manifolds with nonnegative scalar curvature, Geometriae Dedicata, 47 (1993), 255-268, MR 94f:53068, Zbl 0792.53035.

[14] _ Self-duality and four-manifolds with nonnegative curvature on totally isotropic two-planes, Michigan Math. J., 41 (1994), 3-12, MR 95e:53069, Zbl 0816.53023.

[15] _ Positively curved 4-manifolds and the nonnegativity of isotropic curvatures, Michigan Math. J., 44 (1997), 211-229, MR 98d:53055, Zbl 0888.53029.

[16] F. Podestà, Cohomogeneity one Riemannian manifolds and Killing fields, Diff. Geom. Appl., 5 (1995), 311-320, MR 96k:53072, Zbl 0846.53029.

[17] _ Immersions of cohomogeneity one Riemannian manifolds, Monatsh. Math., 122 (1996), 215-225, MR 98a:53067, Zbl 0880.53040.

Received September 29, 2000 and revised March 26, 2001. The first author was partially supported by CNPq, Brasil. The second author was partially supported by FAPESP, Brasil.

\section{IMECC-UNICAMP}

Universidade Estadual de Campinas

13081-970, CAMPINAS

SP, BRASIL

E-mail address: dussan@ime.unicamp.br

Department of Mathematics

California State University Northridge

Northridge, CA, 91330-8313

E-mail address: maria.noronha@csun.edu 